\title{
La confusion : un modèle de syndrome gériatrique !
}

\section{Confusion: a geriatric syndrome pattern!}

\author{
M. Verny \\ (C) Springer-Verlag 2009
}

Ce deuxième numéro des Cahiers de l'année gérontologique aborde dans son dossier thématique le syndrome confusionnel sous différents angles.

Le syndrome confusionnel représente une problématique fréquente et grave en gériatrie justifiant d'aborder ce sujet. La physiopathologie reste en grande partie méconnue et les hypothèses sont multiples autour de défaillance ou d'hyperactivité de neuromédiateurs. Les études aussi bien biologiques qu'en imagerie fonctionnelle restent rares et proposent des résultats discordants. Dans la population âgée, l'hypothèse d'un déficit cholinergique à l'origine de la confusion est probablement l'une des plus séduisantes, même si elle n'est probablement pas exclusive.

C'est en tout cas un excellent exemple de la fragilité des sujets âgés et de la pathologie en cascade. On peut considérer qu'il s'agit d'un modèle de syndrome gériatrique qui nécessite de mettre en œuvre un raisonnement gériatrique rigoureux où l'identification des pathologies chroniques (« facteurs favorisants ») du patient est fondamentale. Sur ce fond, un ou plusieurs facteurs de décompensation (« facteurs déclenchants ») peuvent survenir et être responsables de la survenue du syndrome confusionnel. Une des premières difficultés est l'identification elle-même du syndrome confusionnel qui reste encore très imparfaite, surtout dans les formes hypoactives. C'est dans ces cas que l'utilisation d'outils facilitant le diagnostic comme la Confusion Assessment Method (CAM) est particulièrement utile. L'autre avantage important de ces outils est de permettre «l'éducation » des équipes au repérage de ce syndrome. En pratique, l'élément central à identifier est le trouble attentionnel qui permet de comprendre tous les éléments qui composent le syndrome confusionnel. Dans l'identification de la (ou des) cause(s) de syndrome confusionnel, l'anamnèse est fondamentale avec notamment une enquête très précise des traitements du patient, en portant une

M. Verny $(\bowtie)$

Centre de gériatrie, pavillon M. Bottard, hôpital de La Salpêtrière, 47-83, boulevard de l'hôpital, F-75651 Paris cedex 13, France

e-mail : marc.verny@psl.ap-hop-paris.fr attention toute particulière à ceux introduits récemment ou à l'inverse interrompus (éventuel syndrome de sevrage). Les traitements anticholinergiques sont indiscutablement les plus grands pourvoyeurs de syndrome confusionnel. Au-delà de cette recherche, les causes peuvent aller d'un événement d'apparence banal et peu grave (fécalome, rétention aiguë d'urines, etc.) jusqu'à des pathologies sévères engageant en elle-même le pronostic vital du patient (infarctus myocardique, embolie pulmonaire, etc.). Plus le facteur déclenchant apparait «faible », plus la recherche d'une pathologie favorisante altérant le fonctionnement cognitif devra être acharnée. Tous ces aspects sont détaillés dans l'article de Philippe Chassagne et al.

Ainsi, parmi les affections facilitant l'éclosion d'un syndrome confusionnel, toutes celles responsables d'un syndrome démentiel sont particulièrement concernées. Dans son article, Maxime Godard et al. abordent les frontières ténues existantes entre syndrome démentiel et syndrome confusionnel. Ainsi, bien souvent, la question ne sera pas de savoir s'il s'agit d'un syndrome confusionnel ou démentiel, mais de s'assurer que le syndrome confusionnel ne révèle pas une pathologie démentielle méconnue. Plus difficile, la confusion semble pouvoir révéler dans certains cas une maladie dans une phase prodromale de démence. Quelques études ont pu ainsi montrer que le syndrome confusionnel constituait, en quelque sorte, un facteur de risque de développer une affection démentielle dans les mois ou années suivants. Cela justifie pleinement de la pratique d'un bilan cognitif complet et approfondi à distance de l'épisode confusionnel afin de détecter et prendre en charge le plus précocement possible un syndrome démentiel débutant.

Un aspect tout à fait particulier est la relation entre crises épileptiques et syndrome confusionnel. C'est ce qu'aborde Sandrine Harston. Un premier cas de figure très « classique » est celui de la relation entre la survenue d'une crise généralisée et la constatation d'un syndrome confusionnel postcritique. Dans ces cas, c'est le syndrome confusionnel qui amène à évoquer le diagnostic de crise épileptique. Dans une autre configuration, c'est l'épilepsie qui est directement en cause dans la genèse du syndrome confusionnel. Il s'agit d'une 
manifestation particulière aux sujets âgés : un état de mal non convulsivant dont la seule traduction clinique est la confusion. Il faut savoir y penser puisque, si l'on évoque ce diagnostic, cela amène à la réalisation d'un EEG qui permet le diagnostic dans tous les cas, et donc la mise en œuvre d'un traitement efficace.

Une fois le syndrome confusionnel déclenché, seul le traitement de la (ou des) cause(s) est efficace puisqu'aucun traitement symptomatique n'est actif. Compte tenu des conséquences potentiellement sévères du syndrome confusionnel, l'idéal est de pouvoir l'éviter. Ce sujet est traité par Armelle Tilly-Gentric. Des mesures simples de prise en charge pluridimensionnelle permettent ainsi de réduire l'incidence d'un syndrome confusionnel dans une population âgée à risque. Ces mesures visent les principaux facteurs favorisant comme les troubles neurosensoriels (port de lunettes et/ou des prothèses auditives), la perte des repères spatiotemporels, une mauvaise qualité du sommeil (sans avoir recours à un ou plusieurs traitements psychotropes) ou encore une absence de mobilisation suffisante ou des contentions. La mise en œuvre dans les services de gériatrie de programme de prévention pourrait être considérée comme un des critères de qualités.

L'approche très variée du syndrome confusionnel proposé ici montre à la fois l'importance des connaissances accumulées, le rôle de tous les membres de l'équipe pluridisciplinaire gériatrique et les progrès qu'il y a encore à faire ainsi que toutes les pistes de recherche à poursuivre. Il ne reste plus qu'à se mettre au travail !... 